\title{
Real Time Burning Image Classification Using Support Vector Machine
}

\author{
T.S. Hai ${ }^{1, *}$, L.M. Triet ${ }^{1}$, L.H. Thai ${ }^{2}$, and N.T. Thuy ${ }^{3}$ \\ ${ }^{1} 1$ st Informatics Technology Department, University of Pedagogy, HCMC, Vietnam, \{haits,trietlm\}@ hcmup.edu.vn \\ ${ }^{2}$ 2nd Computer Science Department, University of Science, HCMC, Vietnam, lhthai@ fit.hcmus.edu.vn \\ 33rd University of Engineering and Technology, Ha Noi, Vietnam, nguyenthanhthuy@vnu.edu.vn
}

\section{Abstract}

Burning image classification is critical and attempted problems in medical image processing. This paper has proposed the real time image classification for burning image to automatically identify the degrees of burns in three levels: II, III, and IV. The proposed model uses the multi-colour channels extraction and binary based on adaptive threshold. The proposed model uses One-class Support Vector Machine instead of traditional Support Vector Machine (SVM) because of unbalanced degrees of burns images database. The classifying precision $77.78 \%$ shows the feasibility of our proposed model.

Keywords: burning image classification; Support Vector Machine (SVM); multi-colour channels.

Received on 31 August 2016, accepted on 21 May 2017, published on 06 July 2017

Copyright (0 2017 T.S. Hai et al., licensed to EAI. This is an open access article distributed under the terms of the Creative Commons Attribution licence (http://creativecommons.org/licenses/by/3.0/), which permits unlimited use, distribution and reproduction in any medium so long as the original work is properly cited.

doi: 10.4108/eai.6-7-2017.152760

\section{Introduction}

Medical image processing has a variety of potential applications in the recent years. There are many fields in medical image processing: some emphasize general applicable theory and some focus on specific applications. Computer-aided diagnostic processing has already become an important part of clinical routine. This paper develops the computer aided for burning image classification.

Burns, commonly caused by fire, can also result from chemicals, electricity, and other heat accidents. Burns are classified based on how much of the skin's thickness is involved [1]. The purpose of burning image classification is to automatically identify the degrees of burns based on the colour image of burning patients.

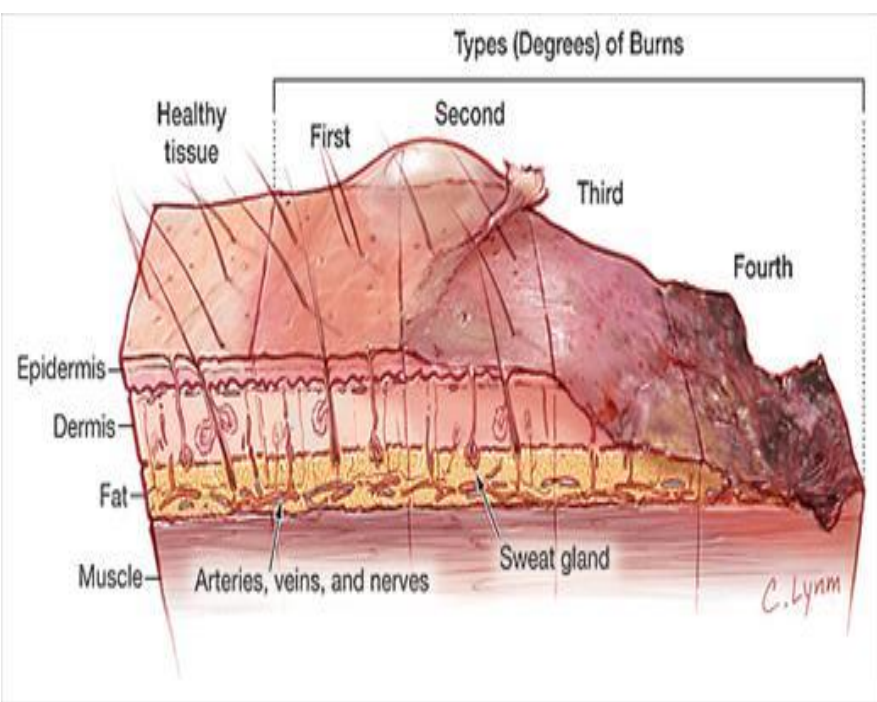

Fig. 1. Degrees of Burns [1] 


\subsection{Medical Image Processing}

The medical imaging is the interesting developing problem

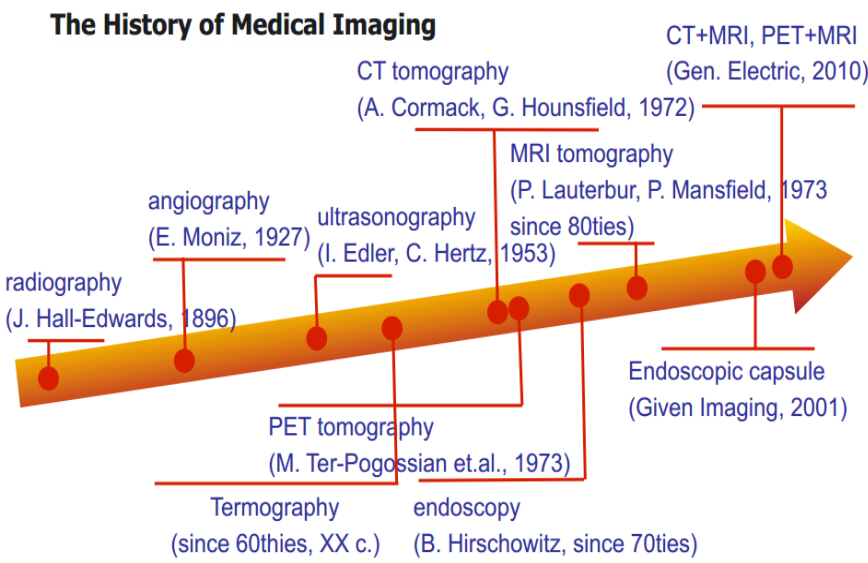

Fig. 2. The history of medical imaging [2]

In recent years, with the developing of computer vision, the computer vision system has been integrated to medical image process system:

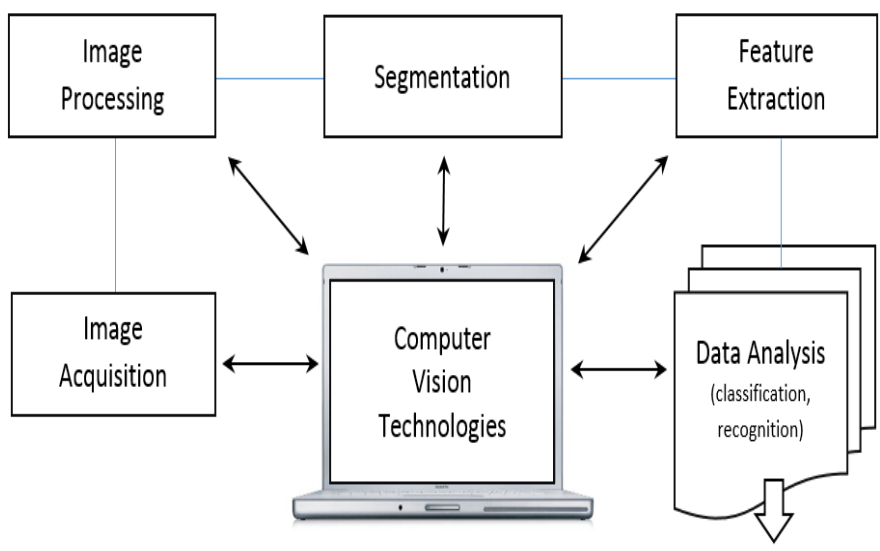

Fig. 3. Computer vision apply for medical image processing

In the above Fig, the computer vision technology can apply for almost phases in the medical imaging system from acquisition, process to feature extraction. Specially, some artificial intelligent tools are very useful for data analysis such as recognition, classification.

In the medical imaging topic, automatic burning image classification is an open problem. In this research, we try to apply computer vision processing and machine learning for identify the degrees of burns. In the clinical burning patient diagnosing code, the doctor need to identify the degrees of burn: Degrees I, Degrees II, Degrees III, and Degrees IV based on the depth of levels of burns and scalds and some clinical diagnosis.

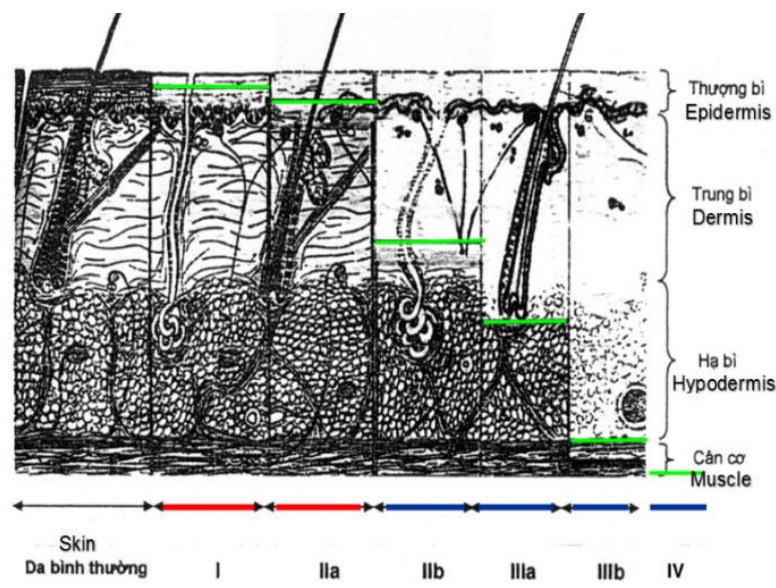

Fig. 4. Different depth levels of burns and scalds $[3,15]$

Degree I of burns involves only the top layer of the skin. Degree II of burns injures deeper into the skin and cause blistering. Degree III of burns involves all the layers of the skin, including the nerves. Degree IV of burns extends into the muscle.

\subsection{Medical Image Processing}

The medical test in the medical diagnosis often uses laboratory analysis, bio signal analysis (ECG, EEEG...) and image analysis. Burning Image Classification is a kind of image classification with the lower number classes in the medical imaging focusing on the image analysis. Thus, we must use the image processing technique in the computer vision for image feature extraction. The feature extraction techniques common used for medical imaging is Fourier transform, 2D function for monochrome image, image brightness profiles, or RGB colour component profiles.

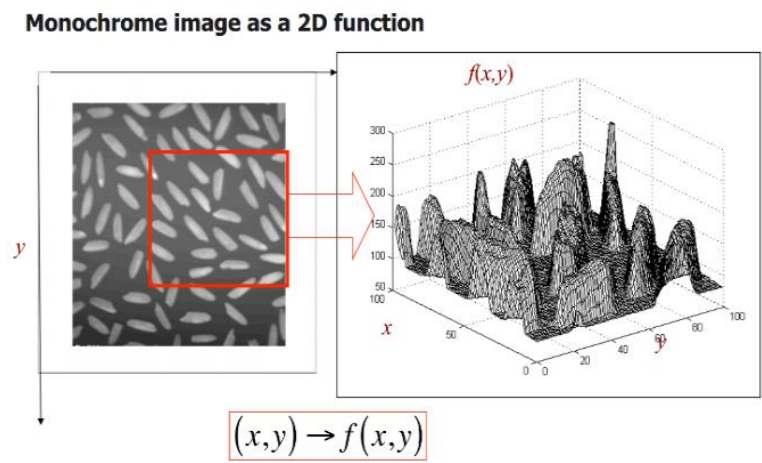

Fig. 5. 2D function transform [2]

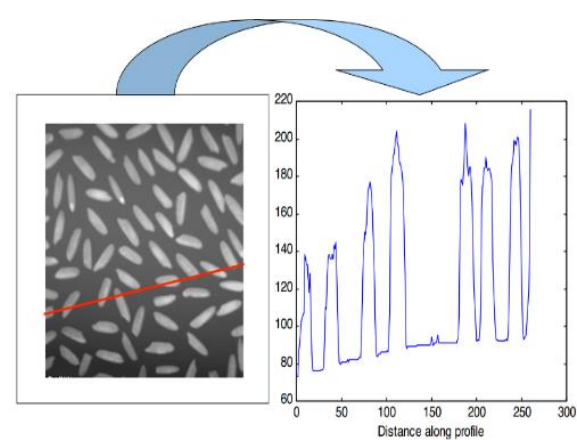

Fig. 6. Image brightness profile [2] 
Fig. 7. An example of multi-color channels
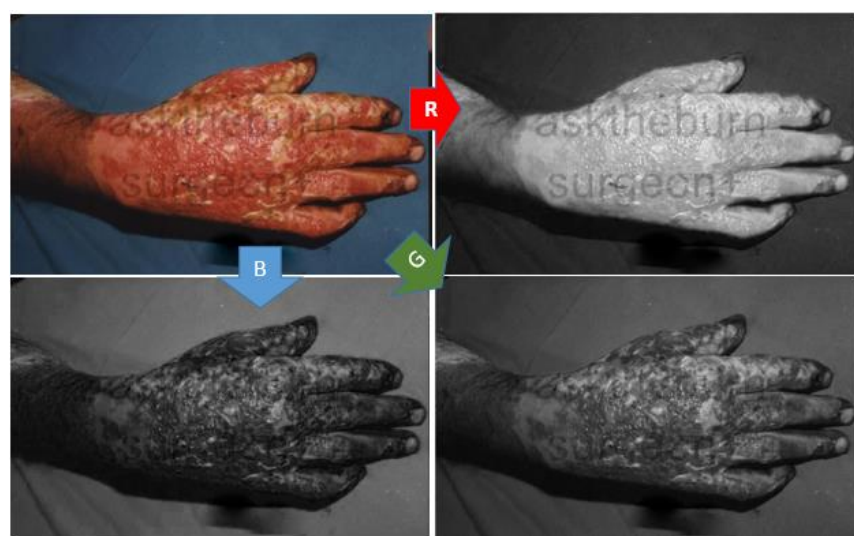

\subsection{Classifier Based on Machine Learning}

The image classification system using machine learning technique often have 2 phases: training phase and classifying phase. In the training phase, the system use machine learning algorithm to update the parameter of classifier model. For example, SVM compute the coefficient of hyper lanes in the training. After that SVM use the hyper lane to classify the image. The common architecture of image classification using machine learning system is below:

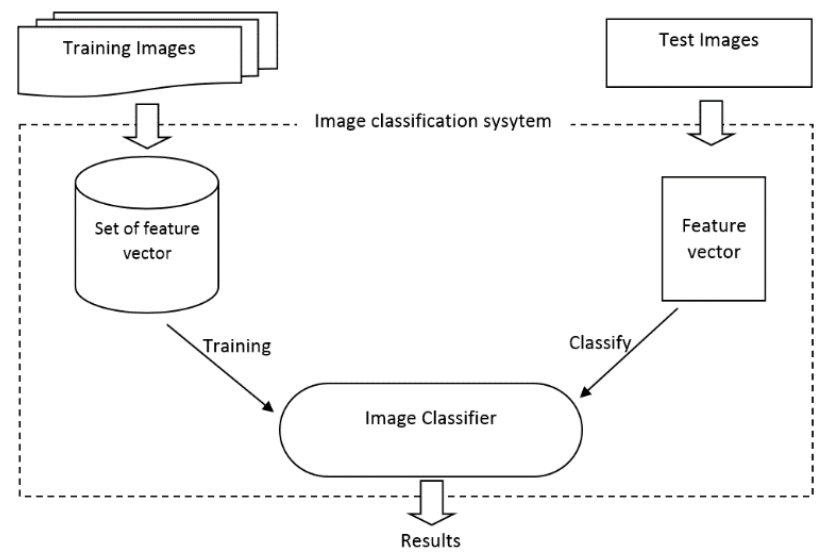

Fig. 8. Image classification architechture using machine learning

There are many kinds of classifiers applied for medical classification: some use expert system and some use learning from data. They often use machine learning Neural Network, Support Vector Machine... with the suitable learning algorithm.

Some popular learning algorithms are unsupervised learning, supervised learning, semi-supervisor learning, or active learning. In the burning image classification, most of burning data from the hospital labelled. Thus, the supervisor learning is the suitable approach.

There is very little burn image classification systems suggested by some researchers [4,9,12]. For example, M. Survana has applied Template Matching, k-NN and SVM classification methods for skin burn images with their own collection dataset with only 120 images in 3 types of burns (superficial dermal, partial thickness and full thickness). This experimental results show that SVM is more suitable classifier for burn images than k-NN and Template Matching.

Besides, B. Acha [5] also proposed the classification of burn wounds using SVM by colour and texture information of burn images. To the best of our knowledge, there is not any image classification system for identifying the degrees of burns based on machine learning in Vietnam. The aim of this paper is to build a real time burning classification applied for Vietnam hospital.

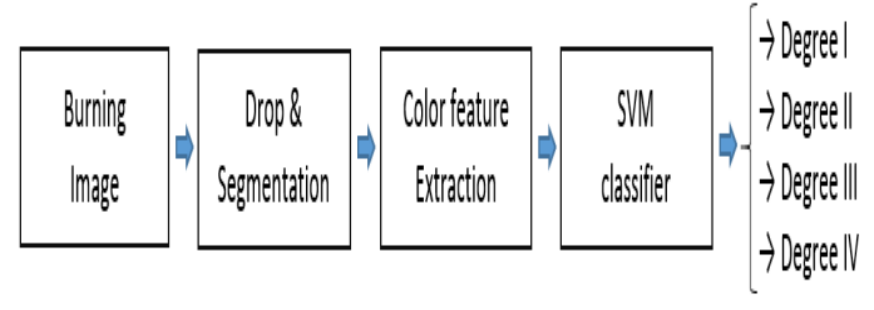

Fig. 9. SVM classifier apply for burning image

The aim of the proposed system will be used as the supplemental resource for the Vietnam doctor's burning diagnose more accurate. The remainder of this paper is organized as follows. In section 2, this paper presents the preprocessing and feature extraction from burning images. In section 3, this paper presents the proposed model applying for burning images classification. The section 4 will present the experimental results and discussion. Conclusion and future works will be mentioned in section 5 .

\section{Burning Image Feature Extraction}

\subsection{Pre-processing}

Firstly, we normalize the burning images in a standard size with the rate $4: 3$.

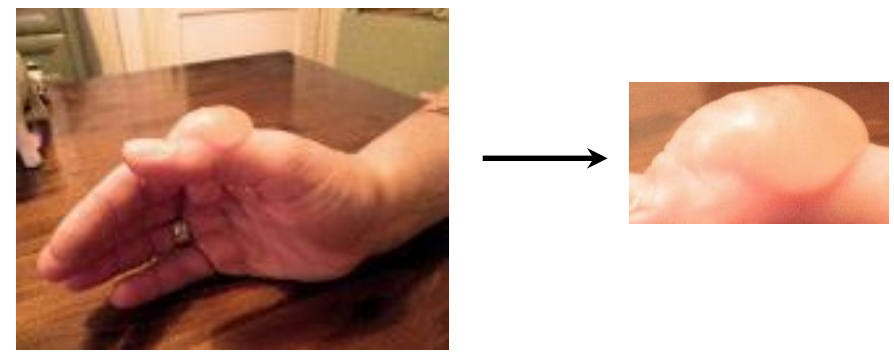

Fig. 10. Burning global standardization

The dropped image will be segmentation using threshold techniques. The frames are the class of Multi Threshold techniques that performs the best in this context $[6,7]$ 

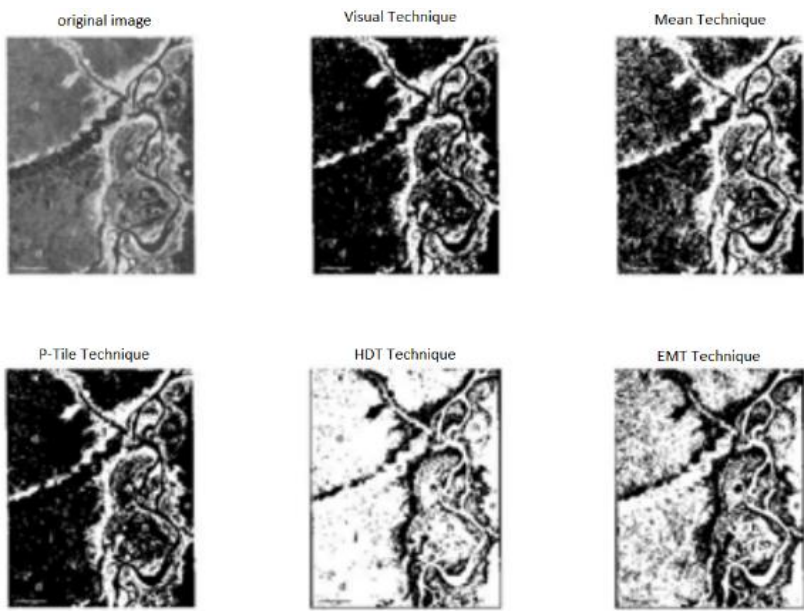

Fig. 11. Threshold techniques [6]

\subsection{Colour Feature Vectors}

Due to the requirement of the processing speed is real time, this paper suggested to use the fast feature extraction based on multi-colour channels Red, Green, Blue and Grey. In order to improve the performance of machine learning, the multi-colour channels will be binary to 0 or 1 .

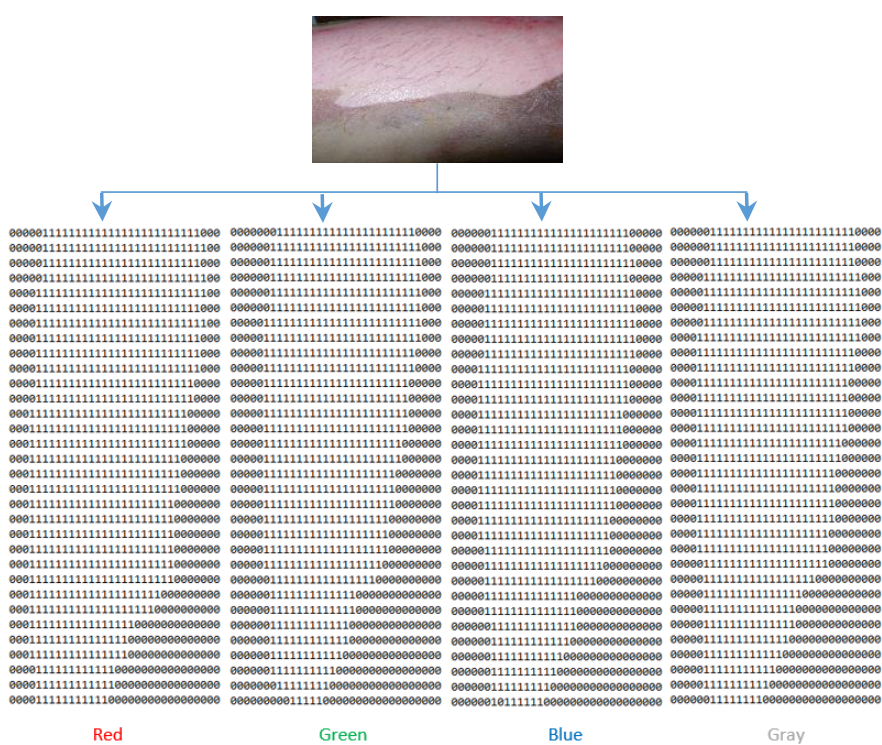

Fig. 12. Multi color channels extraction

\section{Burning Image Classification Model}

The overview of burning image classification model is presented in the below diagram:
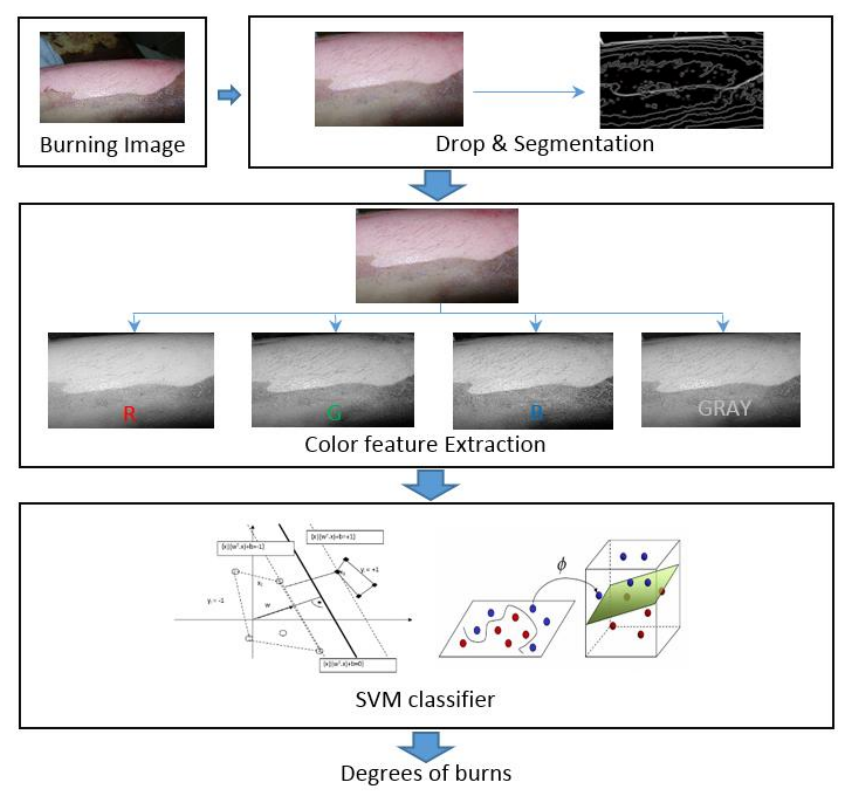

Fig. 13. Burning image classification model

The burning image will be collected and supplied from the Cho Ray hospital, Vietnam. The burning image will be standardize and segmentation before inputting the feature extraction component. The output burning image features are the multi-colour binary channels. The multi-colour channels are the input of one-class SVM classifier.

The goal of SVM classifier is to distinguish test images between a number of classes, using training images. In case, there is a little images of one class if compared to the rest classes. That is the reason why we choose one-class SVM for our burn image classification problem.

\section{Experimental results}

\subsection{Database for Experiment}

We have built image databases from Cho Ray hospital consisting 396 burning images in the 4 degrees of burn II, III and IV. We do not classify the degrees I of burn because it is minor level and might cause from the sun sight. This is also reason why many Vietnamese people do not care about the degrees I of burns at this time

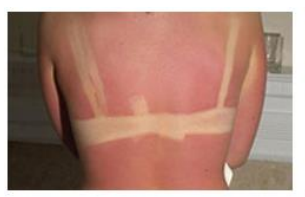

Degree I

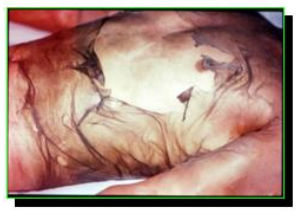

Degree IIIa

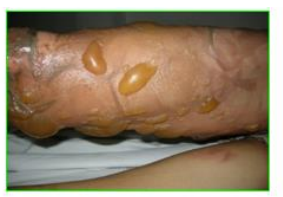

Degree IIa

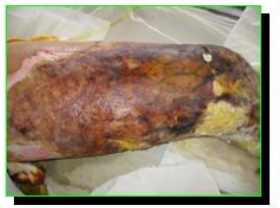

Degree IIIb

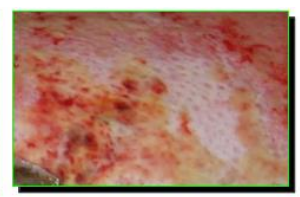

Degree IIb

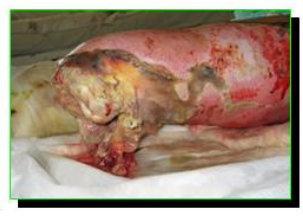

Degree IV

Fig. 14. Illustration for degreses burning image 
The degrees II of burns has 192 images, the degrees III of burns has 180 and the degrees IV of burns has 24 images. There are very little images of the degrees IV of burns because this is high level of burn and the patient may be died before inpatient entrance.

\subsection{Image Storage Organization for \\ Experiment}

To each database, we create two folders: a half images in training and a half images in testing. Figure. 15 shows some images in training folder and Figure. 16 shows some images in testing folder.

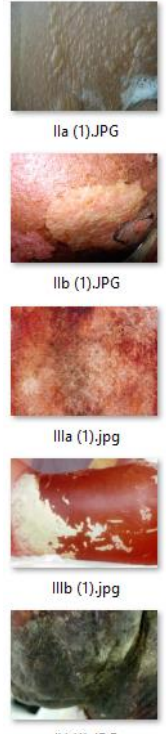

IV (1).JPG

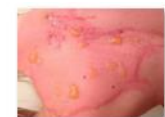

Ila (2).jpg

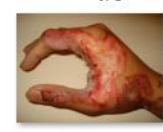

IIb (2).jpg

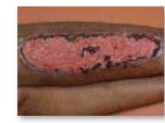

IIIla (2).JPG

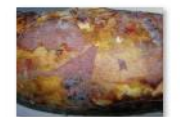

IIIb (2).JPG

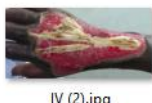

IV (2).jpg

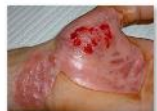

Ila (3).jpg

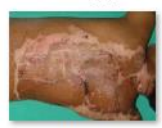

IIb (3).jpg

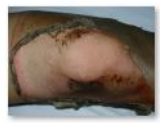

IIIa (3).JPG

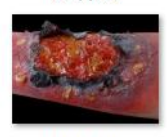

IIIb (3).jpg

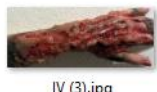

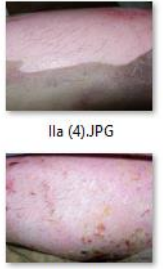

IIb (4).JPG

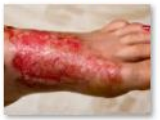

IIIla (4).jpg

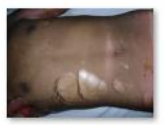

(II) (4) IPG

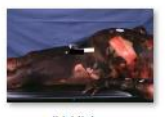

IV (4).jpg

Fig. 15. Some images in training folder.

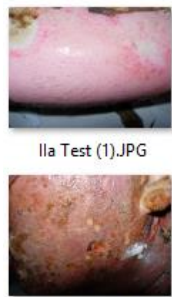

IIb Test (1).JPG

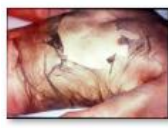

IIIa Test (1).jpg

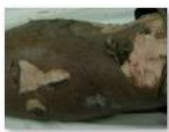

IIIb Test (1).JPG

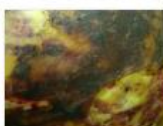

IV Test (1).JPG

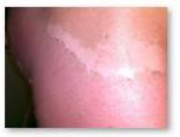

Ila Test (2).jpg

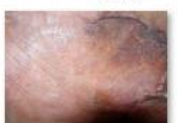

Ilb Test (2).JPG

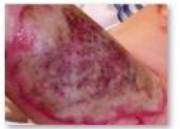

IIla Test (2).jpg

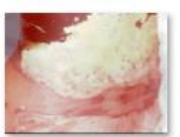

IIIb Test (2).jpg

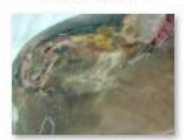

IV Test (2).JPG

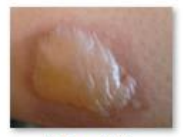

Ila Test (3).jpg

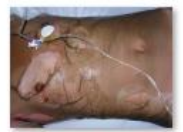

IIb Test (3).JPG

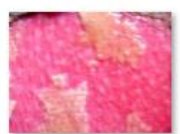

IIla Test (3).JPG

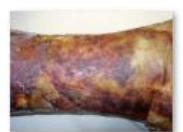

IIIb Test (3).JPG

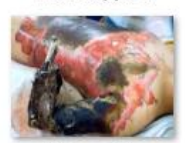

IV Test (3).jpg

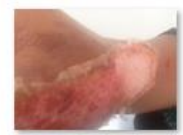

Ila Test (4).jpg

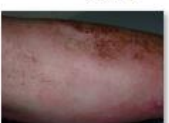

IIb Test (4).JPG

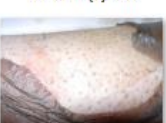

IIla Test (4).JPG
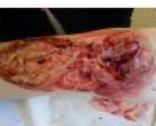

IIIb Test (4).jpg

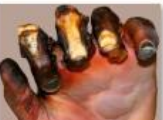

IV Test (4).jpg
The experimental and application have been developed based on Arcord.NET library (http://accord-framework.net/) like image below:

\begin{tabular}{||l|l|l|l|l|||}
\hline \multicolumn{9}{|c|}{ Burning Image Classification Using Support Vector Machine } & \\
\hline
\end{tabular}

Fig. 17. Burn Image Classification User Interface

\subsection{Result of Burning classification}

The precision, false acceptance rate (FAR) and false reject rate is the common criterion to evaluate the classification rate, simplify given by:

$$
\begin{aligned}
\text { Precision } & =\frac{\# \text { correctly classified images }}{\# \text { total images }} \\
F A R & =\frac{\# \text { False classified images }}{\text { classified images }} \\
\text { FRR } & =\frac{\# \text { False rejected images }}{\text { rejected images }}
\end{aligned}
$$

The experimental results are presented in the below tables.

\begin{tabular}{|c|c|c|c|c|c|c|c|}
\hline \multirow{2}{*}{ Degrees } & \multirow{2}{*}{$\begin{array}{l}\text { Number } \\
\text { of } \\
\text { training } \\
\text { images }\end{array}$} & \multirow{2}{*}{$\begin{array}{l}\text { Precision } \\
\text { on } \\
\text { training } \\
\text { images } \\
(\%)\end{array}$} & \multirow{2}{*}{$\begin{array}{l}\text { Number } \\
\text { of } \\
\text { testing } \\
\text { images }\end{array}$} & \multicolumn{2}{|c|}{ Gauss kernel SVM } & \multicolumn{2}{|c|}{$\begin{array}{c}\text { Polynomial kernel } \\
\text { SVM }\end{array}$} \\
\hline & & & & $\begin{array}{l}\text { Right } \\
\text { classified } \\
\text { images }\end{array}$ & $\begin{array}{l}\text { Precision } \\
\quad(\%)\end{array}$ & $\begin{array}{l}\text { Right } \\
\text { classified } \\
\text { images }\end{array}$ & $\begin{array}{l}\text { Precision } \\
\quad \%)\end{array}$ \\
\hline II & 90 & 100 & 90 & 63 & $70 \%$ & 68 & $76 \%$ \\
\hline III & 96 & 100 & 96 & 72 & $75 \%$ & 72 & $75 \%$ \\
\hline IV & 12 & 100 & 12 & 7 & $58 \%$ & 6 & $50 \%$ \\
\hline
\end{tabular}

\section{Table 1. Burning Image Classification Detail Results Using SVM}

Fig. 16. Some images in testing folder. 


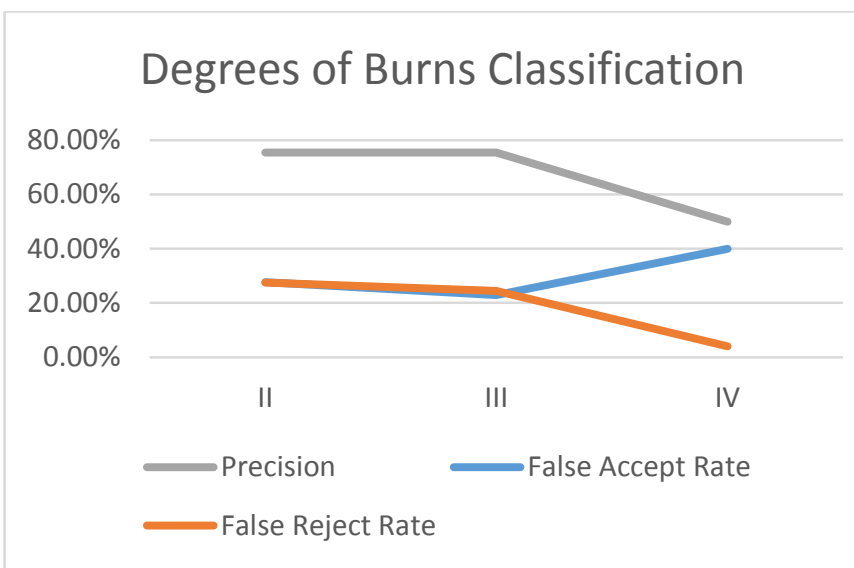

Fig. 18. Precision and FAR- FRR Degrees of Burn Identification results

In the above Figure. 18, the FAR of Degrees IV is high and the FRR of Degrees IV is low because of unbalanced burning image of Degrees IV image is too low due to the number of burn inpatient in this level.

Table 2. Classification Results Using Gauss And Polynomial Kernel SVM

\begin{tabular}{|llllll|}
\hline & \multicolumn{5}{c}{$\begin{array}{l}\text { \#right classified } \\
\text { images }\end{array}$} \\
\cline { 3 - 6 } \#training & \#testing & & Precision $(\%)$ \\
images & images & Gauss & Polynomial & Gauss & Polynomial \\
& & Kernel & Kernel & Kernel & Kernel \\
& & SVM & SVM & SVM & SVM \\
\hline 198 & 198 & 142 & 144 & $71.71 \%$ & $73.73 \%$ \\
\hline
\end{tabular}

Table 3. Classification Results using One-Class SVM vs. SVM

\begin{tabular}{|llll|}
\hline & & & \\
\#training & \#testing & \#right classified & Precision $(\%)$ \\
images & images & & \\
\hline 198 & 198 & 154 & $77.78 \%$ \\
\hline
\end{tabular}

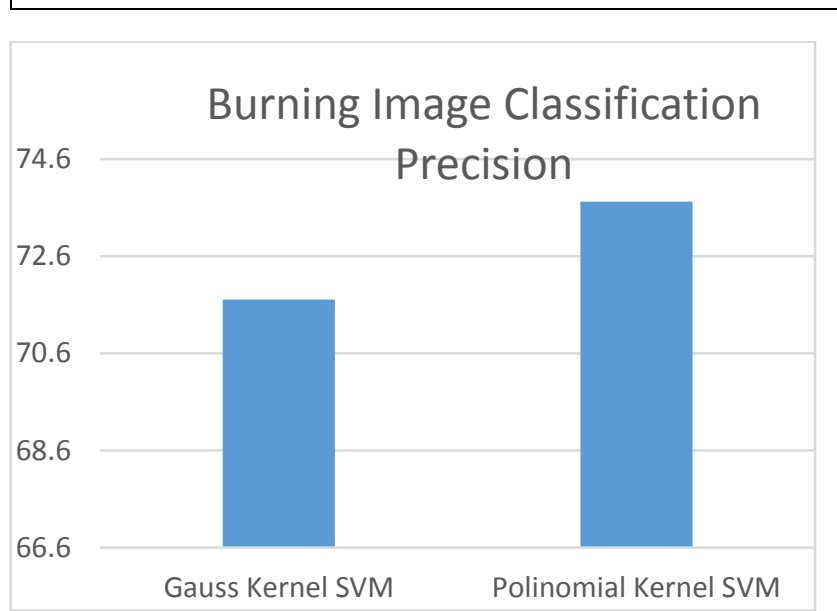

Fig. 19. Burning Image Classification Results using kernelbased SVM

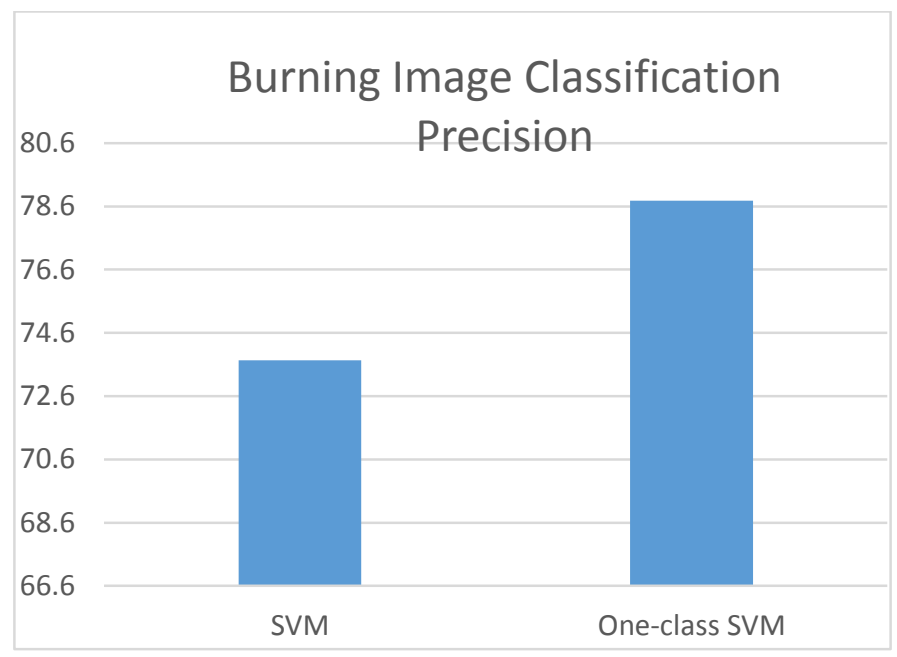

Fig. 20. One-class SVM vs. SVM

The above Figure. 20 show that the One-Class SVM (OC$\mathrm{SVM}$ ) is suitable in this classification problem because of the unbalanced data $[8,10]$. OC-SVM has improved the accuracy of burn classification. However, OC-SVM required the trade of computing time to the accuracy in the case using the simple binary features. Therefore, the process time may be not in real time, but it may be still acceptable for the easy in the real time processing time standard in the Vietnam government (see more details on the Vietnamese government site http://mic.gov.vn/).

The experiments of classification time with fixed 3 classed (Degree II, Degree III, and Degree IV of burn level) and on 3 binary colour channels, in the normal PC computer of Dr in Cho Ray hospital, is presented in the table.

Table 4. Classification Time

\begin{tabular}{|c|c|c|c|}
\hline $\begin{array}{l}\text { \#No. of } \\
\text { processing } \\
\text { images }\end{array}$ & $\begin{array}{l}\text { \#classification } \\
\text { time (mm:ss) }\end{array}$ & \#No. of classes & $\begin{array}{l}\text { \#No. of binary } \\
\text { colour channel }\end{array}$ \\
\hline 0 & 0 & 3 & 3 \\
\hline 80 & $00: 58.8274598$ & 3 & 3 \\
\hline 160 & $01: 57.3859362$ & 3 & 3 \\
\hline 240 & 03:41.6291760 & 3 & 3 \\
\hline 320 & $08: 20.2476083$ & 3 & 3 \\
\hline 396 & $14: 10.8322941$ & 3 & 3 \\
\hline
\end{tabular}

In the worst experimental condition, the process time is less than 3 seconds per case. It is acceptable for real time application in Vietnam government hospital.

\subsection{Discussion:}

From experimental results of SVM classification method using Polynomial kernel is more accuracy than Gauss kernel. It maybe cause from the distributed of burning image data is distributed $48 \%$ in the degrees II of burn and $47 \%$ in the degrees III of burns. The rest is distributed under $5 \%$.

The wrong classification is focus on the Degrees IV of burn. The number of images of this degree is too lower than the Degrees II and III. Due to this unbalance in the training, 
we try to use one-class SVM instead of traditional SVM. It has been improve the accuracy of classification.

\section{Conclusion and Future Works}

This paper has proposed the real time image classification for burning image to identify automatically the degrees of burns.

The proposed model uses the multi-colour channels extraction and binary based on adaptive threshold. In order to the system can be process in the real time performance in Vietnam condition. Even though, the contribution of this paper is the application of one-class SVM to the burning image classification problem, and there is no theoretical contribution in this manuscript. The proposed method for the burning image classification can be used as the supplemental resource for diagnose burn level by Vietnamese doctors more accurate result.

Because of the unbalance degrees of burns data, we suggest use one-class SVM instead of traditional SVM. The experimental results show the feasibility of the proposed model in the starting researching phase. The expectation of improving the accuracy and the real time processing are opening challenges of this problem.

We cannot trade off the classification time because its requirement is real time in the diagnosis degrees of burns phase of treatment process. However, we can trade off the training time to improve the precision of classification. So that in the future work, we can use a big data for training phase and use some improved SVM $[11,12,14]$ such Fuzzy SVM or another complex training model in order to increase the classifying accuracy.

\section{Acknowledgement}

We would like to gratefully and sincerely thank Doctor Vo Van Phuc and his colleges in the burn department of Cho Ray hospital for his helping, guidance, understanding, and most importantly, his expertise during this study.

\section{References}

[1] Torpy, Janet M., Cassio Lynm, and Richard M. Glass. "Burn injuries." JAMA302.16 (2009): 1828-1828.

[2] Michał Strzelecki, "Medical Image: Introduction to Medical Imaging", Biomedical Engineering, IFE, 2013

[3] Zellweger G., Die behandlung der verbrennungen, Deutscher XizteVerlag, Kohl, 1985.

[4] Suvarna, Malini, and U. C. Niranjan. "Classification Methods of Skin Burn Images." International Journal of Computer Science \& Information Technology 5.1 (2013): 109.

[5] Begoña Acha ; Carmen Serrano ; Jose' I. Acha and Laura M. Roa "Segmentation and classification of burn images by color and texture information", J. Biomed. Opt. 10(3), 034014 (May 11, 2005). ;http://dx.doi.org/10.1117/1.1921227.

[6] Al-Amri, Salem Saleh, and Namdeo V. Kalyankar. "Image segmentation by using threshold techniques." arXiv preprint arXiv:1005.4020 (2010).

[7] Chebira, Amina, and Jelena Kovačević. "Multiresolution techniques for the classification of bioimage and biometric datasets." Optical Engineering+ Applications. International Society for Optics and Photonics, 2007.

[8] Guerbai, Yasmine, Youcef Chibani, and Bilal Hadjadji. "The effective use of the one-class SVM classifier for handwritten signature verification based on writer-independent parameters." Pattern Recognition 48.1 (2015): 103-113.
[9] Coelho, Luis Pedro, et al. "Principles of bioimage informatics: focus on machine learning of cell patterns." Linking Literature, Information, and Knowledge for Biology. Springer Berlin Heidelberg, 2010. 8-18.

[10] Le Hoang Thai, Hai, Tran Son, and Nguyen Thanh Thuy. "Image classification using support vector machine and artificial neural network." International Journal of Information Technology and Computer Science (IJITCS) 4.5 (2012): 32.

[11] Lu, Dengsheng, and Qihao Weng. "A survey of image classification methods and techniques for improving classification performance." International journal of Remote sensing 28.5 (2007): 823-870.

[12] Spadotto, Marcelo M., et al. "Classification of burn degrees in grinding by neural nets." The IASTED International Conference on Artificial Intelligence and Applications, Innsbruck, Austria. 2008.

[13] Duda, Richard O., Peter E. Hart, and David G. Stork. Pattern classification. John Wiley \& Sons, 2012.

[14] Le, Thai Hoang, Hai Son Tran, and Thuy Thanh Nguyen. "Applying Multi Support Vector Machine for Flower Image Classification." Context-Aware Systems and Applications. Springer Berlin Heidelberg, 2012. 268-281.

[15] Bệnh viện Chợ Rẫy, Phỏng, Sổ tay hướng dẫn lâm sàng, Tài liệu lưu hành nội bộ, TP HCM, trang 632-641, 2005 (Vietnamese).

[16] Hai, Tran Son, Thai, Le Hoang and Nguyen Thanh Thuy. "Facial expression classification using artificial neural network and k-nearest neighbor." International Journal of Information Technology and Computer Science (IJITCS) 7.3 (2015): 27. 